\title{
Applying a real-coded multi-population genetic algorithm to multi-reservoir operation
}

\author{
L. Chen ${ }^{1 *}$ and F. J. Chang ${ }^{2}$ \\ ${ }^{1}$ Department of Civil Engineering, Chung Hua University, Hsin Chu, Taiwan, ROC \\ 2 Department of Bioenvironmental systems Engineering and Hydrotech Research Institute, National Taiwan University, Taipei, Taiwan, ROC
}

\begin{abstract}
:
The primary objective of this study is to propose a real-coded hypercubic distributed genetic algorithm (HDGA) for optimizing reservoir operation system. A conventional genetic algorithm (GA) is often trapped into local optimums during the optimization procedure. To prevent premature convergence and to obtain near-global optimal solutions, the HDGA is designed to have various subpopulations that are processed using separate and parallel GAs. The hypercubic topology with a small diameter spreads good solutions rapidly throughout all of the subpopulations, and a migration mechanism, which exchanges chromosomes among the subpopulations, exchanges information during the joint optimization to maintain diversity and thus avoid a systematic premature convergence toward a single local optimum. Three genetic operators, i.e. linear ranking selection, blend- $\alpha$ crossover and Gaussian mutation, are applied to search for the optimal reservoir releases. First, a benchmark problem, the four-reservoir operation system, is considered to investigate the applicability and effectiveness of the proposed approach. The results show that the known global optimal solution can be effectively and stably achieved by the HDGA. The HDGA is then applied in the planning of a multi-reservoir system in northern Taiwan, considering a water reservoir development scenario to the year 2021. The results searched by an HDGA minimize the water deficit of this reservoir system and provide much better performance than the conventional GA in terms of obtaining lower values of the objective function and avoiding local optimal solutions. Copyright (c) 2006 John Wiley \& Sons, Ltd.
\end{abstract}

KEY WORDS genetic algorithm; premature convergence; subpopulations; multi-reservoir system

Received 15 December 2004; Accepted 8 November 2005

\section{INTRODUCTION}

Modelling a multi-reservoir system, especially a large one, is a challenging task for a system analyst (Wurbs, 1993; Jairaj and Vedula, 2000). A reservoir-systemanalysis model usually contains a great number of complex mechanisms for making release decisions in each period within a framework of user-specified operating rules and/or criteria functions. Typical reservoir constraints include conservation (continuity) equations, maximum and minimum storages, maximum and minimum releases, penstock and equipment limitations, and contract with legal and institutional obligations. As stated by Bower et al. (1962), the performance of the system as simulated (or optimized) at the design stage must correspond closely to that attainable after the system is built. Determining the optimal release schedule of a reservoir system for planning purposes has been an important area of research. After the aims have been determined, most problems can be solved by techniques developed in the fields of operations research and management sciences (Yeh, 1985). Mujumdar and Narulkar (1992) briefly reviewed the various methods available for modelling multi-reservoir systems. Traditional approaches, including linear programming (LP), nonlinear programming

* Correspondence to: L. Chen, Department of Civil Engineering, Chung Hua University, Hsin Chu, Taiwan, ROC. E-mail: lichen@chu.edu.tw and dynamic programming (DP), for solving large-scale mathematical programs applied to reservoir operations involve decomposition, partitioning, aggregation, and various successive approximation techniques.

Genetic algorithms (GAs) originated in the mid 1970s (Holland, 1975) have been developed into a powerful optimization approach. Goldberg (1989) and Michalewicz (1992) give excellent introductions to GAs, and several recent papers present summaries of the essentials (e.g. Wardlaw and Sharif, 1999). A principal difference between optimization using a GA versus more traditional methods is that the decision space is searched from an entire population of potential designs. This difference enables GAs to solve discrete, non-convex, discontinuous problems without differentiation (Goldberg, 1989). The GAs are iterative procedures evolving a population of individuals that are candidate solutions to a specific domain. During each generation, the individuals in the current population are rated for their effective evaluations, and a new population of candidate solutions is formed using specific genetic operators, such as selection, crossover, and mutation (Grefenstette, 1986). The cycle of evolution is repeated until a desired termination criterion is reached. Goldberg (1989) and Davis (1991) reviewed many important applications of GAs.

One of the most serious failure modes for a GA is premature convergence, which leads to the search becoming trapped in a region that does not contain the global 
optimum (Eshelman and Schaffer, 1991). This problem was previously tackled by several different methods. For example, Renders and Flasse (1996), hybridized a GA with hill-climbing methods, such as the quasi-Newton and Nelder-Mead's simplex. A similar strategy was also presented by Muhlenbein et al. (1991), where local search procedures were integrated to the GA. In this study, a diversity preservation method based on spatial separation is used to avoid the premature convergence of a conventional GA. A distributed GA could partition the population into several subpopulations, each one of them being processed by individual GAs. Thus, each subpopulation is treated as a separate breeding unit under the control of a conventional GA (Davidor, 1991; Collins, 1992). Furthermore, a migration mechanism produces a chromosome exchange between the subpopulations to encourage the proliferation of good genetic material throughout the whole population; and this is easy to implement on parallel hardware (Herrera and Lozano, 1997). Therefore, this GA can be fully exploited in its parallel structure to gain the required speed for practical uses.

This study employs a hypercubic distributed GA (HDGA) for optimizing multi-reservoir operation schemes. The next section characterizes in detail the basic properties of a real-coded HDGA. The third section describes the implementation, including topology and migration of the HDGA. A benchmark problem, the fourreservoir problem, solved by the HDGA, is then presented in the fourth section and these results are compared with those obtained by a conventional GA. We implement the HDGA method to search the optimal operation scheme of a multi-reservoir system in Taiwan in the penultimate section, and draw conclusions in the final section.

\section{BASICS OF A HYPERCUBIC DISTRIBUTED GENETIC ALGORITHM}

\section{Conventional real-coded genetic algorithm}

A growing number of researchers in the GA community have come to champion large-alphabet real-coded (or floating-point) genes as opposed to small-alphabet binarycoded genes. Although few theorists believe that small alphabets should be more effective than large alphabets (Antonisses, 1989; Wright, 1991), practical experience shows that real-coded genes with their large alphabet work better (Davis, 1991). According to a couple of our previous studies, real-coded GAs have advantages over binary-coded GAs in the operation of a reservoir system (Chang and Chen, 1998; Chang et al., 2005). Hence, in this study, we only consider real-coded GAs. For a real-coded GA, a chromosome corresponds to a vector of real parameters, a gene corresponds to a real number, and an allele corresponds to a real value. By doing this, each population member is represented by a chromosome, which is the parameter vector $x=\left(x_{1}, x_{2}, \ldots x_{m}\right) \in R^{m}$.

\section{Selection mechanism}

The selection mechanism is an important GA operator with responsibility for the diversity of the population.
It may maintain or eliminate diversity, depending on its current selective pressure (Herrera and Lozano, 1997). A linear ranking selection (Baker, 1985) provides a nice and effective alternative way for an HDGA, since its selective pressure could easily be adjusted by means of varying an associated control parameter. The chromosomes are sorted in order of raw fitness (the value of the objective function), and then the selection probability of each chromosome $C_{i}$ is computed according to its rank by using the following function:

$$
E\left(C_{i}\right)=\eta_{\max }-\left(\eta_{\max }-\eta_{\min }\right) \frac{\operatorname{rank}\left(C_{i}\right)-1}{N-1}
$$

where $N$ is the population size, $\eta_{\min } \in[0,1]$ specifies the expected number of copies for the worst chromosome, and $\eta_{\max }=2-\eta_{\min }$ is the expected number of copies of the best chromosome; the rank of the best $C_{i}$ is 1 , the rank of the second best $C_{i}$ is 2 , and the rank of the worst $C_{i}$ is $N$, as shown in Figure 1 .

Linear ranking will go with stochastic universal sampling (Goldberg and Deb, 1991). This procedure guarantees that the number of copies of any chromosome is bounded by the floor and ceiling of its expected number of copies.

\section{Real crossover}

Crossover is a technique that takes two parent chromosomes and produces two child chromosomes. The conventional binary-coded GA uses a crossover operator that exchanges arbitrary substrings between two individuals with probability $P_{\mathrm{c}}$. The crossover operator of the realcoded GA mimics the $k$-point crossover of a binary-coded GA, which considers offspring as some perturbations from the values of their parents.

Blend crossover (BLX- $\alpha$ ) uniformly picks values that lie between two points that contain the two parents, but may extend equally on either side determined by a userspecified GA parameter $\alpha$ (see Figure 2). For example, BLX-0.5 picks parameter values from points that lie on an interval that extends $0.5 I$ on either side of the interval $I$ between the parents. The BLX- 0.5 is less likely to

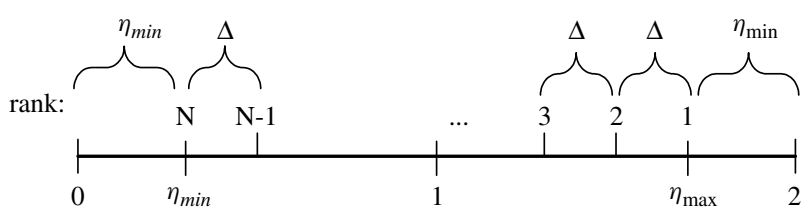

Figure 1. Expected number of copies (from $\eta_{\min }$ to $\eta_{\max }$ with uniform displacement $\left.\Delta=\left(2-\eta_{\min }\right) /(N-1)\right)$

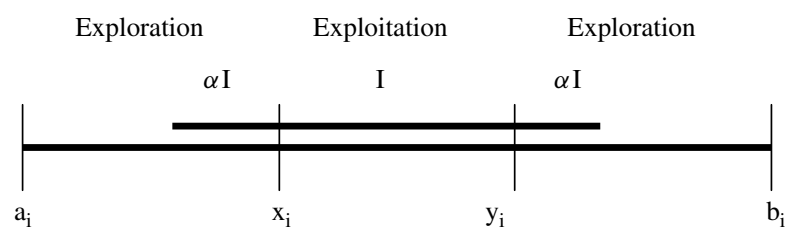

Figure 2. BLX crossover 
converge prematurely from empirical tests (Chang and Chen, 1998).

\section{Real mutation}

Mutation is used for finding new points in the search space. In binary-coded GA, mutation is the occasional random alteration, with small probability, of the value of a string position. Recently, Gaussian mutation together with arithmetical crossover has usually been used to produce offspring for the real-coded GA. A Gaussian mutation operator (Hinterding, 1995) requires two parameters: the mean, which is often set to zero, and the standard deviation $\sigma$, which can be interpreted as the mutation step size. Mutations then are realized by replacing components of the vector $x$ by

$$
x_{i}^{\prime}=x_{i}+N(0, \sigma)
$$

where $N(0, \sigma)$ is a random Gaussian number with mean zero and standard deviation.

\section{Algorithm with parallel structure}

A conventional GA is likely to be trapped in a region that does not contain the global optimum. This problem, called premature convergence, has been recognized as a serious failure mode for GAs (Eshelman and Schaffer, 1991). A multi-population GA divides a single population into smaller subpopulations. Each subpopulation evolves by genetic operations in parallel with the other, while maintaining a limited but powerful interaction between all subpopulations. The most important advantage of subpopulations is the enhanced diversity among the subpopulations. Therefore, subpopulations that are made to evolve in an autonomous manner, but not completely independently, explore different domains of the search space and avoid a possible loss of diversity by exchanging information (Karpouzos et al., 2001).

Topology is an important factor in the performance of the distributed GAs because it determines the speed at which a good solution spreads to other subpopulations. If the topology has a dense connectivity, or a short diameter, or both, good solutions will spread quickly to all of the subpopulations (Cantu-Paz, 1995). There are several different topologies to achieve a distributed GA. Figure 3

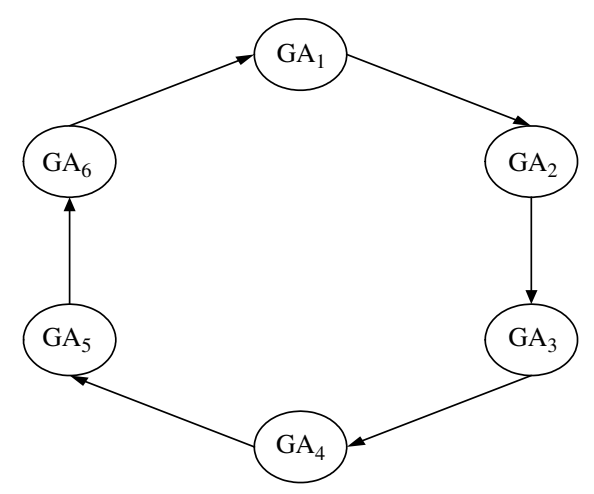

Figure 3. Ring migration

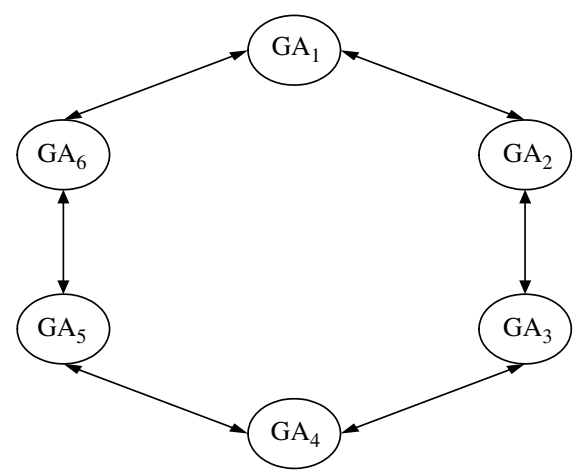

Figure 4. Neighbourhood migration

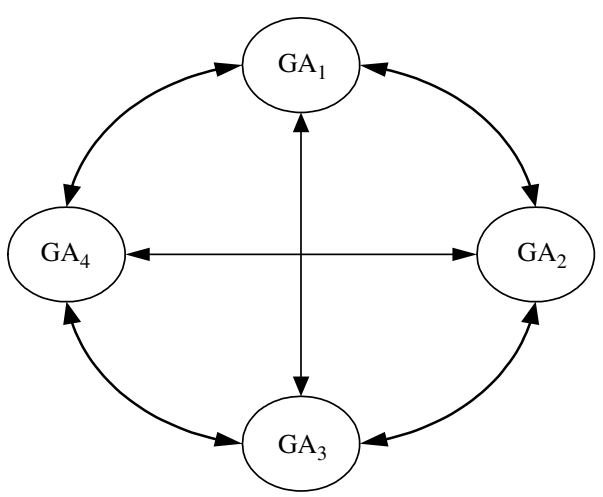

Figure 5. Unrestricted migration

shows the ring topology, where individuals are transferred between directionally adjacent subpopulations. A similar strategy, known as neighbourhood, is shown in Figure 4, where migration can be made between nearest neighbours bidirectionally. The unrestricted migration formulation is depicted in Figure 5, where individuals may migrate from one subpopulation to another (Man et al., 1999). Tanese $(1987,1989)$ and Erickson et al. (1991) presented a parallel GA implemented on a hypercube structure. The topology of neighbourhood is better than that of a ring because of the former can transfer the good solution in two directions with a faster speed. Whereas, the unrestricted topology has too dense a connection to lead to premature convergence during the optimizing processes. Therefore, the topology of the hypercube presented in this paper with a proper diameter can maintain a good balance between computing speed and diversity of the genetic materials.

\section{Application of genetic algorithms for reservoir operation}

GAs have been used successfully for a number of engineering design applications within water resources (Goldberg, 2000). In the reservoir operation system optimization fields, GAs have been demonstrated as powerful optimization approaches. Esat and Hall (1994) applied a GA to the four-reservoir problem. They concluded that GAs have potential in water resources optimization and that significant savings could be achieved in memory and execution times. Olivera and Loucks (1997) used GAs to develop operating policies for multi-reservoir systems, 
and concluded that GAs are practical and robust methods that could lead to effective operating policies. Chang and Chen (1998) applied a real-coded GA for rule-based flood control reservoir management. The results show that the real-coded GA performed better in terms of efficiency and precision than the binary-coded GA. Wardlaw and Sharif (1999) demonstrated an assumed four-reservoir deterministic problem using GAs; the results show that GAs provided robust and acceptable solutions. Recently, Sharif and Wardlaw (2000) presented multi-reservoir systems optimization using GAs. They compared their GA optimization with discrete differential DP and showed not only that GA results are very close to the optimum, but the technique also appears to be robust. Cai et al. (2001) deal with global versus local optimal solutions in reservoir operation. They applied a GA and LP approach to two nonlinear models; the results indicated that, for larger instances, the GA and LP approach finds solutions with significantly better objective values. Chen (2003) applied a real-coded GA for the optimization of a longterm reservoir and indicated that the real-coded GA with several revised operators significantly improved the performance of the system. Chang et al. (2003) used a GA to optimize reservoir operation rule curves and flushing schedule in a reservoir. The real-coded GA in the context of reservoir systems optimization is not limited by the type of the objective function. Unlike many traditional optimization algorithms, there is no requirement for the objective function to be differentiable or continuous. Besides, a significant advantage of the GA approach is that no initial trial release policy is required (Chen, 2003). Therefore, the real-coded GA is used as a basic approach to develop a parallel structure for the optimization of reservoir operation in this study.

\section{IMPLEMENTATION OF THE HYPERCUBIC DISTRIBUTED GENETIC ALGORITHM}

The HDGA with three dimensions presented in this paper is shown in Figure 6. A detailed presentation of this algorithm can be found in Herrera and Lozano (2000). The major difference between an HDGA and a conventional GA is the parallel structure of the HDGA; each subpopulation uses an independent conventional GA with different crossover parameters. These algorithms represent a promising way for introducing a correct exploration/exploitation balance in order to avoid premature convergence and reach approximate final solutions.

It includes two important different sides. The front side is devoted to exploration. There are four subpopulations $E_{1}, \ldots, E_{4}$, to which exploratory crossover operators are applied. The exploratory degree increases clockwise, starting at the lowest $E_{1}$ and ending at the highest $E_{4}$. The other side (the rear side) is for exploitation. There are four subpopulations $e_{1}, \ldots, e_{4}$, and exploitative crossover operators are used. The exploitation degree increases clockwise, starting at the lowest $e_{1}$ and finishing at the highest $e_{4}$. With this structure, a parallel multi-resolution

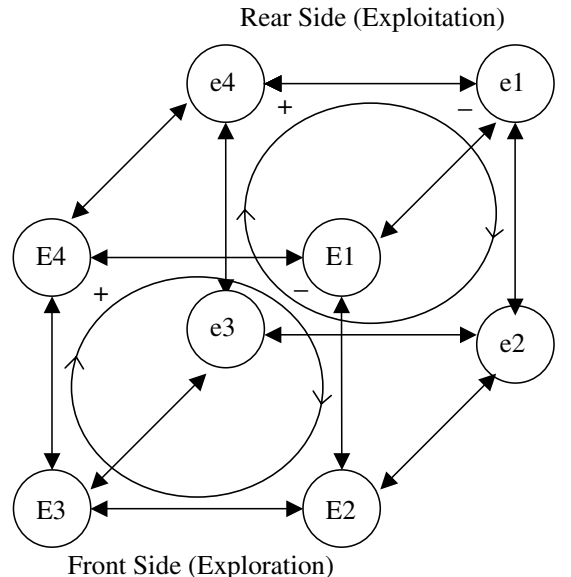

(a) Basic topology

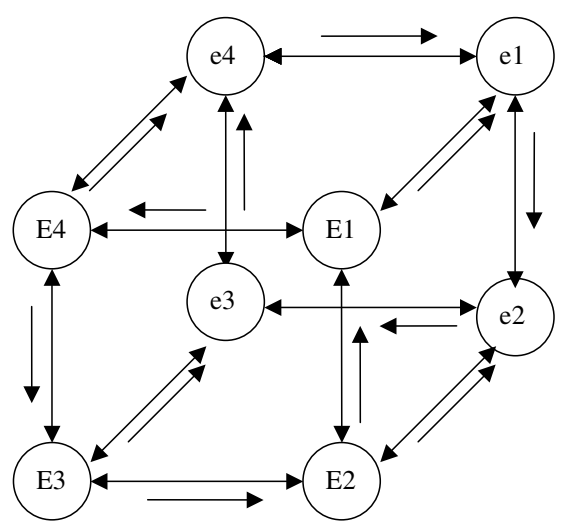

(b) Refinement

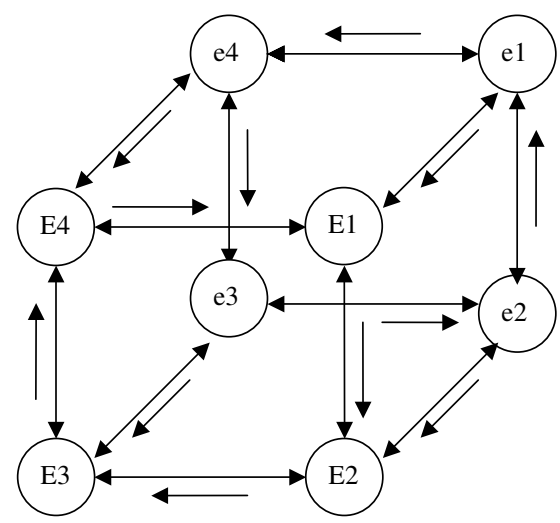

(c) Expansion

Figure 6. Structure of an HDGA: (a) basic topology; (b) refinement; (c) expansion

is obtained by using the crossover operation, which allows a diversified search (reliability) and an effective local tuning (accuracy) to be achieved simultaneously (Figure 6a).

\section{Refinement and expansion}

In order to improve the reliability and accuracy, two effects are introduced. The first effect is 'refinement' (Figure 6b), which makes migrations form an exploratory subpopulation toward an exploitative one, i.e. from $E_{\mathrm{i}}$ to $e_{i}$, or between two exploratory subpopulations from a 
higher degree to a lower one, i.e. from $E_{i+1}$ to $E_{i}$, or between two exploitative subpopulations from a lower degree to a higher one, i.e. from $e_{i}$ to $e_{i+1}$. The second effect is 'expansion' (Figure 6c), which makes migrations in the opposite direction.

\section{Migration}

An emigration model is one in which migrants are sent only toward immediate neighbours along a dimension of the hypercube, and each subsequent migration takes place along a different dimension of the hypercube. In particular, the best individual of each subpopulation is sent toward the corresponding subpopulation every five generations (Herrera and Lozano, 2000), as shown in Figure 7. The sequence of application is from left to right: i.e. first, the refinement migrations; second, the refinement/expansion migrations; third, the expansion migrations; and then the sequence starts again. In this way, the best elements (emigrants) do not affect the same subpopulation for a long time, which would probably occur using the elitist strategy of a conventional GA. Finally, with this migration schema, a global elitist strategy persists, since the best element of all subpopulations is never lost, although it is moved from one subpopulation to another.

\section{Parameters setting}

The $\eta_{\min }$ of linear ranking selection in Equation (1) assigned for the subpopulations of the HDGA is shown in Table I. This indicates that the more exploratory an $E_{i}$ subpopulation is, the higher the selective pressure it will undergo. In contrast, the more exploitative an $e_{i}$ subpopulation is, the less selective pressure it will undergo.

The $\alpha$ values of blend- $\alpha$ crossover are also listed in Table I. The function of adjusting $\alpha$ is to achieve the exploratory or exploitative degree of different subpopulations. Only when $\alpha=0.5$ is there a balanced relationship reached between convergence (exploitation) and divergence (exploration); the probability that a gene will lie in the exploitation interval is then equal to the probability that it will lie in an exploration interval (Eshelman and Schaffer, 1993). In the absence of selection pressure, all values $\alpha<0.5$ will demonstrate a tendency for the population to converge toward values in the centre of their ranges, producing low diversity levels in the population, and inducing a possible premature convergence toward nonoptimal solutions.

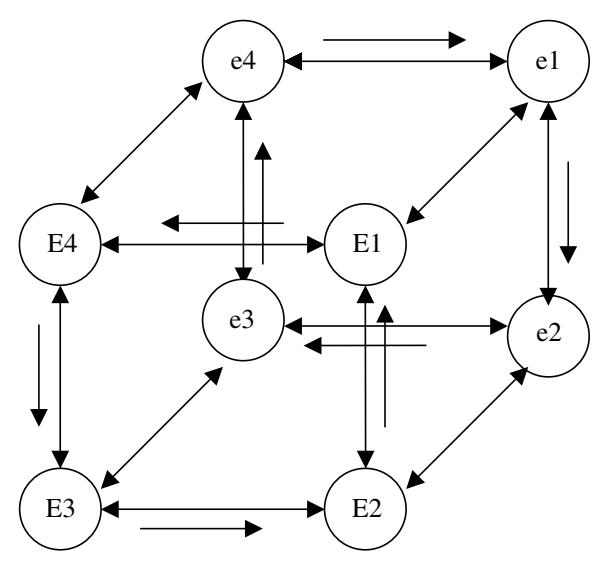

(a) REFINEMENT MIGRATIONS

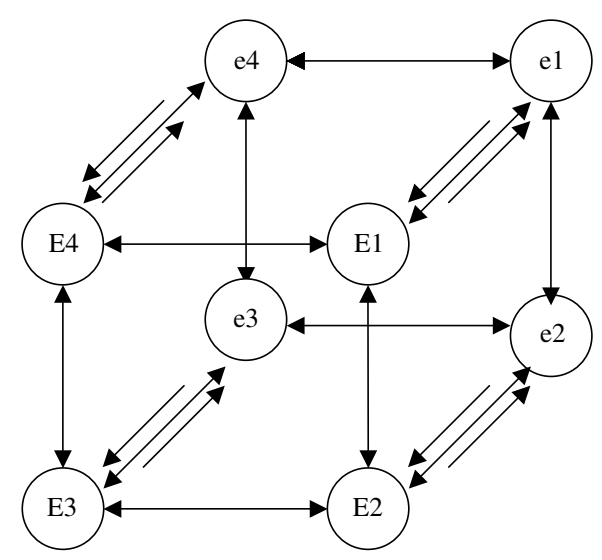

(b) REF/EXP MIGRATIONS

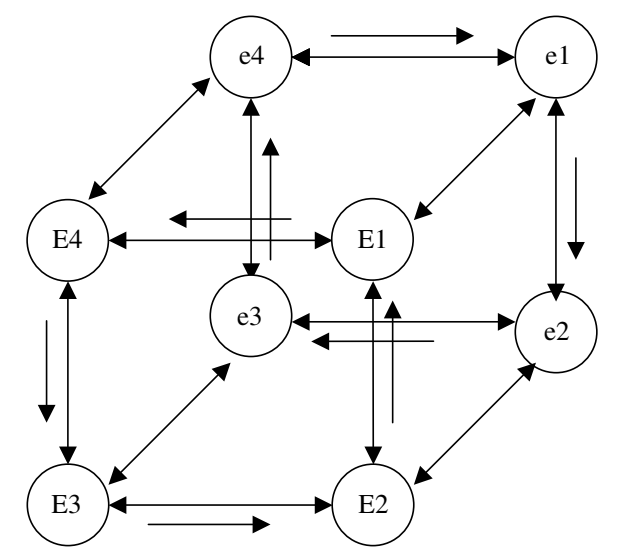

(c) EXPANSION MIGRATIONS

Figure 7. Three types of migration in an HDGA: (a) refinement migrations; (b) ref/exp migrations; (c) expansion migrations

Table I. The $\eta_{\min }$ and $\alpha$ values for each subpopulation

\begin{tabular}{|c|c|c|c|c|c|c|c|c|}
\hline \multirow[t]{3}{*}{ Parameters } & \multicolumn{4}{|c|}{ Crossover exploitation } & \multicolumn{4}{|c|}{ Crossover exploration } \\
\hline & \multicolumn{2}{|c|}{+} & \multicolumn{2}{|c|}{-} & \multicolumn{2}{|c|}{-} & \multicolumn{2}{|c|}{+} \\
\hline & $e_{4}$ & $e_{3}$ & $e_{2}$ & $e_{1}$ & $E_{1}$ & $E_{2}$ & $E_{3}$ & $E_{4}$ \\
\hline$\eta_{\text {min }}$ & $0 \cdot 8$ & 0.7 & $0 \cdot 6$ & $0 \cdot 5$ & $0 \cdot 3$ & $0 \cdot 2$ & $0 \cdot 1$ & $0 \cdot 0$ \\
\hline$\alpha$ & $0 \cdot 1$ & $0 \cdot 2$ & $0 \cdot 3$ & $0 \cdot 4$ & $0 \cdot 5$ & 0.6 & 0.7 & $0 \cdot 8$ \\
\hline
\end{tabular}




\section{A BENCHMARK PROBLEM}

\section{System definitions}

The four-reservoir problem was first formulated and solved by Larson (1968), and more recently by Esat and Hall (1994) and Wardlaw and Sharif (1999). This problem offered an opportunity to test the performance of GAs against a known global optimum (Wardlaw and Sharif, 1999).

The system consists of four reservoirs, as shown in Figure 8 . The objective is to maximize benefits, including hydropower generation and irrigation, from the system over 12 operating periods of $2 \mathrm{~h}$. The initial storage in all reservoirs is set as five units, and the inflows to the first and second reservoirs are two units and three units respectively for all 12 periods.

The fundamental constraints on reservoir storage are

$$
\begin{gathered}
0 \cdot 0 \leq S_{1}, S_{2}, S_{3} \leq 10 \\
0 \cdot 0 \leq S_{4} \leq 15
\end{gathered}
$$

And the capacities of water release from reservoirs through the turbines are

$$
\begin{gathered}
0 \cdot 0 \leq R_{1} \leq 3 \\
0 \cdot 0 \leq R_{2}, R_{3} \leq 4 \\
0 \cdot 0 \leq R_{4} \leq 7
\end{gathered}
$$

The above constraints apply in all time steps. The continuity constraints for each reservoir over each operating period $t$ are

$$
S_{i}(t+1)=S_{i}(t)+I_{i}(t)+M R_{i}(t)
$$

where $S_{i}(t)$ is the vector of reservoir storage at time $t$ in the reservoirs $i=1,4, I_{i}(t)$ is the vector of reservoir inflows in time period $t$ to the reservoirs $i=1,4, R_{i}(t)$

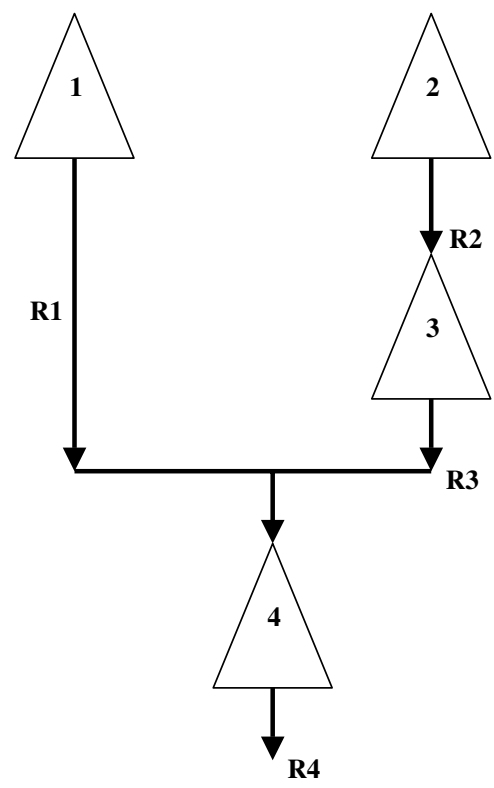

Figure 8. Four-reservoir problem is the vector of reservoir releases in time period $t$ from reservoirs $i=1,4$, and $M$ is a $4 \times 4$ matrix of indices of reservoir connection:

$$
\left[\begin{array}{cccc}
-1 & 0 & 0 & 0 \\
0 & -1 & 0 & 0 \\
0 & 1 & -1 & 0 \\
1 & 0 & 1 & -1
\end{array}\right]
$$

In addition to the above general constraints, there are final target storages for all reservoirs. There are five units for reservoirs 1 to 3 , and seven units for reservoir 4 . The objective function to be maximized can be written as

$$
\operatorname{Max}(I)=\sum_{i=1}^{4} \sum_{t=1}^{12} b_{i}(t) R_{i}(t)+\sum_{t=1}^{12} b_{5}(t) R_{4}(t)
$$

The benefit functions $b_{i}(t)$, tabulated by Larson (1968) and by Heidari et al. (1971), are shown in Table II. For comparative purposes, taking the target ending storage in reservoir $i$ to be $d_{i}$, the penalty function is expressed as

$$
g_{i}\left[S_{i}(12), d_{i}\right]=-40\left[S_{i}(12)-d_{i}\right]^{2} \text { for } S_{i}(12) \leq d_{i}
$$

and

$$
g_{i}\left[S_{i}(12), d_{i}\right]=0 \quad \text { for } S_{i}(12)>d_{i}
$$

According to Wardlaw and Sharif (1999), the objective function is thus modified to

$$
\begin{aligned}
\operatorname{Max}(I)= & \sum_{i=1}^{4} \sum_{t=1}^{12} b_{i}(t) R_{i}(t)+\sum_{t=1}^{12} b_{5}(t) R_{4}(t) \\
& +\sum_{i=1}^{4} g_{i}\left[S_{i}(12), d_{i}\right]
\end{aligned}
$$

\begin{tabular}{|c|c|c|c|c|c|}
\hline$n$ & $b_{1}(n)$ & $b_{2}(n)$ & $b_{3}(n)$ & $b_{4}(n)$ & $b_{5}(n)$ \\
\hline 1 & $1 \cdot 1$ & 1.4 & 1.0 & 1.0 & 1.6 \\
\hline 2 & 1.0 & $1 \cdot 1$ & 1.0 & $1 \cdot 2$ & 1.7 \\
\hline 3 & 1.0 & 1.0 & 1.2 & 1.8 & 1.8 \\
\hline 4 & 1.2 & 1.0 & 1.8 & $2 \cdot 5$ & 1.9 \\
\hline 5 & 1.8 & $1 \cdot 2$ & $2 \cdot 5$ & $2 \cdot 2$ & $2 \cdot 0$ \\
\hline 6 & $2 \cdot 5$ & $1 \cdot 8$ & $2 \cdot 2$ & $2 \cdot 0$ & $2 \cdot 0$ \\
\hline 7 & $2 \cdot 2$ & 2.5 & $2 \cdot 0$ & $1 \cdot 8$ & $2 \cdot 0$ \\
\hline 8 & $2 \cdot 0$ & $2 \cdot 2$ & 1.8 & $2 \cdot 2$ & 1.9 \\
\hline 9 & 1.8 & $2 \cdot 0$ & $2 \cdot 2$ & 1.8 & 1.8 \\
\hline 10 & $2 \cdot 2$ & 1.8 & $1 \cdot 8$ & 1.4 & 1.7 \\
\hline 11 & 1.8 & $2 \cdot 2$ & 1.4 & $1 \cdot 1$ & 1.6 \\
\hline 12 & 1.4 & 1.8 & $1 \cdot 1$ & 1.0 & 1.5 \\
\hline
\end{tabular}

The known global optimum for the four-reservoir problem is $401 \cdot 3$.

\section{Results and discussions}

The conventional genetic algorithm. To solve the fourreservoir problem by using a GA, it is necessary to construct a chromosome representing releases in all four reservoirs in all 12 time steps. The chromosome length (genes) may be expressed as a product of the

Table II. The benefits of the four-reservoir problem 
number of stages and number of reservoirs in the system. For this problem, we had 48 genes $(12 \times$ 4), and we set the population size as 100 and ran with a fixed length of 1000 generations. Three genetic operators are used: linear ranking selection with $\eta_{\min }=$ 0.4 , blend $\alpha=0.5$ crossover, and the Gaussian mutation of real-coded representation. The results demonstrate that the conventional GA exhibits a slower rate of improvement in fitness in the mid-generation period of the run, but it sustains its rate of improvement longer. The maximum value of the objective function achieved after 500 generations with real-value coding equals $400 \cdot 5$, which is a suboptimal solution that the conventional GA converged to prematurely.

The hypercubic distributed genetic algorithm. Each subpopulation size is set to be 50 and the parameters of $\eta_{\min }$ and $\alpha$ are shown in Table I. The other parameters are the same as the conventional GA mentioned above. In the final generation, the best objective value is found to be $401 \cdot 3$, which is the same as the known global optimal solution. One of the optimal solutions of this problem by using the HDGA is shown in Table III. The

Table III. One of the optimal solutions of the four-reservoir problem

\begin{tabular}{lrrrrrrrrrrrrr}
\hline Time & 0 & 1 & 2 & 3 & 4 & 5 & 6 & 7 & 8 & 9 & 10 & 11 & 12 \\
\hline Storage 1 & 5 & 6 & 8 & 10 & 10 & 9 & 8 & 7 & 6 & 5 & 4 & 3 & 5 \\
Release 1 & 1 & 0 & 0 & 2 & 3 & 3 & 3 & 3 & 3 & 3 & 3 & 0 & \\
Storage 2 & 5 & 4 & 6 & 7 & 10 & 10 & 9 & 8 & 7 & 6 & 7 & 6 & 5 \\
Release 2 & 4 & 1 & 2 & 0 & 3 & 4 & 4 & 4 & 4 & 2 & 4 & 4 & \\
Storage 3 & 5 & 9 & 10 & 8 & 4 & 3 & 3 & 3 & 3 & 3 & 1 & 1 & 5 \\
Release 3 & 0 & 0 & 4 & 4 & 4 & 4 & 4 & 4 & 4 & 4 & 4 & 0 & \\
Sorage 4 & 5 & 6 & 4 & 1 & 0 & 0 & 0 & 0 & 0 & 0 & 0 & 7 & 7 \\
Release 4 & 0 & 2 & 7 & 7 & 7 & 7 & 7 & 7 & 7 & 7 & 0 & 0 & \\
\hline
\end{tabular}

optimal (best) fitness was found at the end of each generation. The on-line performance is used as the performance measure. The on-line behaviours of eight subpopulations are demonstrated in Table IV. It appears that all subpopulations are converging to the global optimal solution within 200 generations. The optimal solutions of exploration subpopulations $E_{1}, \ldots, E_{4}$ are more diverse than the exploitation subpopulations $e_{1}, \ldots, e_{4}$ in the early stages. In the later stages, most subpopulations are converged because of migrations. Clearly, the HDGA could solve this reservoir operation problem more efficiently than a conventional GA does.

Moreover, the optimal solution is found to be not unique. The alternative optimal solution can be obtained by changing the releases of reservoirs 2 and 3, which are two sequential reservoirs, and their benefit functions $b_{i}(t)$ multiplied by the release $R_{i}(t)$ at certain time step could produce the same benefit.

\section{A REAL-WORLD CASE STUDY IN TAIWAN}

The average annual rainfall in Taiwan is high, i.e. $2500 \mathrm{~mm}$, compared with the world average of $970 \mathrm{~mm}$. However, over $75 \%$ of the annual rainfall occurs during the wet season. Typhoons usually occur in June to September, bringing much of the needed rainfall for the coming dry season, during which an enormous amount of water must be provided for irrigating rice paddies. Such a significant seasonal variation in annual rainfall makes reservoir operation complicated. The Tan-Shui River basin reservoirs system is our case study to evaluate the performance of the HDGA. These reservoirs (in northern Taiwan) are the most important source of water supply for irrigation and domestic use (around 6 million residents); several hydraulic power plants are also supplied from this system. Hence, optimizing the operations of this multireservoir system is crucial and complex.

Table IV. The on-line performance of eight subpolulations

\begin{tabular}{|c|c|c|c|c|c|c|c|c|}
\hline Generation & $e_{1}$ & $e_{2}$ & $e_{3}$ & $e_{4}$ & $E_{1}$ & $E_{2}$ & $E_{3}$ & $E_{4}$ \\
\hline 10 & $379 \cdot 3$ & $377 \cdot 3$ & $378 \cdot 5$ & $378 \cdot 6$ & $370 \cdot 3$ & $371 \cdot 4$ & $369 \cdot 1$ & $368 \cdot 3$ \\
\hline 20 & $385 \cdot 6$ & $385 \cdot 2$ & $385 \cdot 5$ & 385.9 & $384 \cdot 2$ & $384 \cdot 3$ & $386 \cdot 4$ & $382 \cdot 3$ \\
\hline 30 & $391 \cdot 6$ & $391 \cdot 5$ & $391 \cdot 1$ & $391 \cdot 2$ & 389.8 & $390 \cdot 6$ & 391 & 389.9 \\
\hline 40 & 395.4 & 394.7 & 395 & 395.4 & $394 \cdot 3$ & 393.8 & 394.9 & $394 \cdot 2$ \\
\hline 50 & $397 \cdot 6$ & $397 \cdot 4$ & $397 \cdot 7$ & $397 \cdot 5$ & $397 \cdot 3$ & $397 \cdot 1$ & $397 \cdot 1$ & 397 \\
\hline 60 & 399 & $398 \cdot 7$ & 398.9 & $398 \cdot 8$ & 398.9 & 398.9 & $398 \cdot 2$ & $398 \cdot 3$ \\
\hline 70 & $399 \cdot 5$ & $399 \cdot 1$ & 399.7 & $399 \cdot 6$ & $399 \cdot 5$ & $399 \cdot 2$ & $399 \cdot 3$ & $399 \cdot 6$ \\
\hline 80 & 399.9 & $399 \cdot 7$ & 400 & $400 \cdot 2$ & 399.8 & $400 \cdot 1$ & $400 \cdot 3$ & 400 \\
\hline 90 & $400 \cdot 6$ & $400 \cdot 3$ & $400 \cdot 3$ & $400 \cdot 6$ & $400 \cdot 3$ & $400 \cdot 3$ & $400 \cdot 3$ & $400 \cdot 2$ \\
\hline 100 & $400 \cdot 8$ & $400 \cdot 6$ & $400 \cdot 8$ & $400 \cdot 7$ & $400 \cdot 6$ & $400 \cdot 7$ & $400 \cdot 7$ & $400 \cdot 4$ \\
\hline 110 & 401 & $400 \cdot 8$ & $400 \cdot 9$ & 401 & $400 \cdot 8$ & $400 \cdot 9$ & $400 \cdot 7$ & 401 \\
\hline 120 & $401 \cdot 1$ & $400 \cdot 9$ & $400 \cdot 9$ & $401 \cdot 1$ & $401 \cdot 1$ & $400 \cdot 9$ & $400 \cdot 9$ & 401 \\
\hline 130 & $401 \cdot 1$ & $401 \cdot 1$ & $401 \cdot 1$ & $401 \cdot 1$ & $401 \cdot 1$ & $401 \cdot 1$ & 401 & 401 \\
\hline 140 & $401 \cdot 1$ & $401 \cdot 1$ & $401 \cdot 1$ & $401 \cdot 1$ & $401 \cdot 1$ & $401 \cdot 2$ & $401 \cdot 1$ & $401 \cdot 1$ \\
\hline 150 & $401 \cdot 1$ & $401 \cdot 1$ & $401 \cdot 1$ & $401 \cdot 1$ & $401 \cdot 1$ & $401 \cdot 2$ & $401 \cdot 2$ & $401 \cdot 1$ \\
\hline 160 & $401 \cdot 3$ & $401 \cdot 2$ & $401 \cdot 3$ & $401 \cdot 3$ & $401 \cdot 1$ & $401 \cdot 3$ & $401 \cdot 2$ & $401 \cdot 1$ \\
\hline 170 & $401 \cdot 3$ & $401 \cdot 2$ & $401 \cdot 3$ & $401 \cdot 3$ & $401 \cdot 1$ & $401 \cdot 3$ & $401 \cdot 3$ & $401 \cdot 1$ \\
\hline 180 & $401 \cdot 3$ & $401 \cdot 3$ & $401 \cdot 3$ & $401 \cdot 3$ & $401 \cdot 1$ & $401 \cdot 3$ & $401 \cdot 3$ & $401 \cdot 2$ \\
\hline 190 & $401 \cdot 3$ & $401 \cdot 3$ & $401 \cdot 3$ & $401 \cdot 3$ & $401 \cdot 3$ & $401 \cdot 3$ & $401 \cdot 3$ & $401 \cdot 2$ \\
\hline 200 & $401 \cdot 3$ & $401 \cdot 3$ & $401 \cdot 3$ & $401 \cdot 3$ & $401 \cdot 3$ & $401 \cdot 3$ & $401 \cdot 3$ & $401 \cdot 3$ \\
\hline
\end{tabular}




\section{System description}

The drainage area of the Tan-Shui River basin is $2762 \mathrm{~km}^{2}$ and has two major parallel storage reservoirs, i.e. the Shih-Men and Fei-Tsui reservoirs. The growing industrial and urban development in the basin over the last 50 years has drastically increased the water demand. Both present and future water demands in the basin will be met primarily by using surface water sources. Various development schemes have been considered to improve the utilization of available water resources. Among these, the most likely plan is the construction of the KaoTai reservoir (see Figure 9). Combined with the original two reservoirs, there are three reservoirs in this water supply network system. Because the drought in the ShihMen area is more serious than in the Fei-Tsui area, the Shih-Men reservoir system with the most serious drought periods (from June 1964 to May 1965) is chosen as a scenario in this paper. The detailed descriptions of these reservoirs are as follows.

Shih-Men reservoir. Shih-Men reservoir, which was completed in 1964 and now has a storage capacity of $250.02 \times 10^{6} \mathrm{~m}^{3}$, was built primarily for irrigation and water supply along the Ta-Han River. The hydropower plant at Shih-Men has a generating capacity of $45 \mathrm{MW}$. There are three irrigation areas and several public water plants in the water supply system that use the water released from this reservoir.

Fei-Tsui reservoir. Fei-Tsui reservoir was completed in 1985 and now has a storage capacity of $390.2 \times 10^{6} \mathrm{~m}^{3}$.

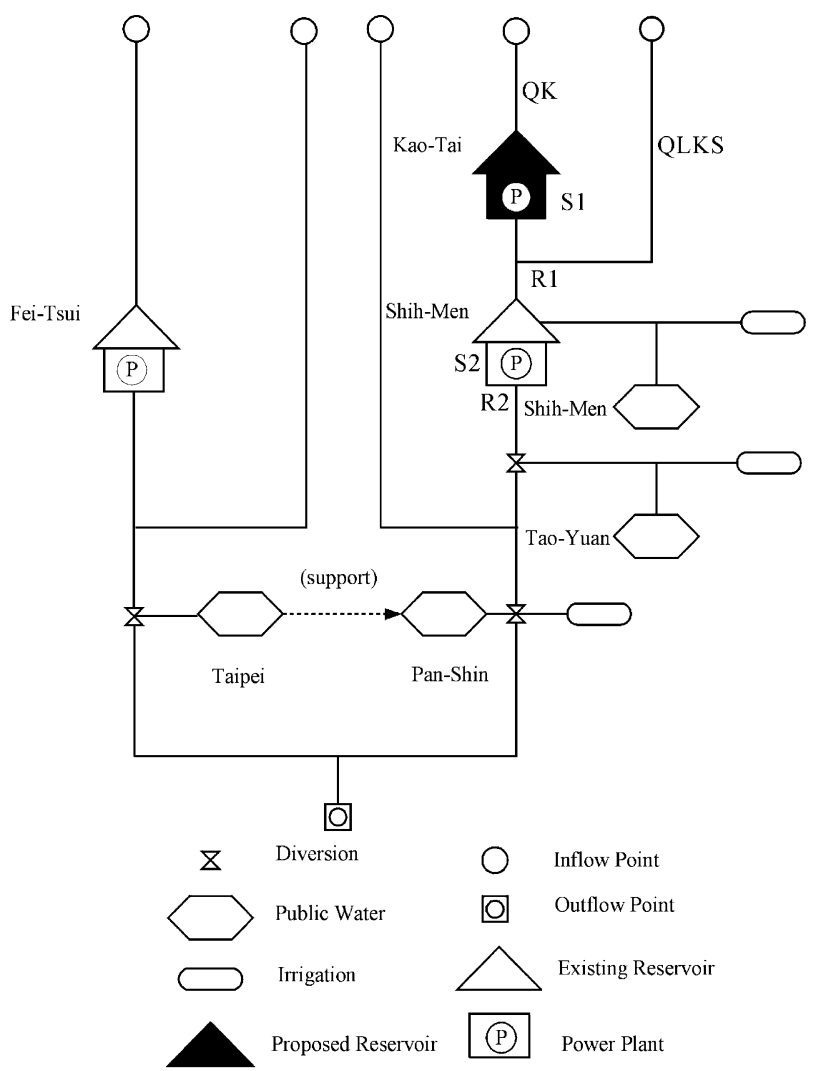

Figure 9. Schematic diagram of Tan-Shui River basin
The hydropower plant at Fei-Tsui has a generating capacity of $70 \mathrm{MW}$. The primary water use in the basin is potable for water demand to the city of Taipei. The function of this reservoir here is simplified to support the fixed amount of public water targets of the Pan-Shin plant.

Future development scenario. Kao-Tai reservoir will be constructed upstream of the Shih-Men reservoir. These two reservoirs are typical serial reservoirs in Ta-Han Stream, Tan-Shui River basin. The main purposes of Kao-Tai reservoir include water supply and hydropower generation. The storage capacity is $211.3 \times 10^{6} \mathrm{~m}^{3}$. In order to evaluate the function of Kao-Tai reservoir, two cases, i.e. one excluding (case 1) and one including (case 2) Kao-Tai reservoir, are considered and compared.

\section{Input data}

Reservoir properties. The main properties, i.e. the heights of the dams, the storage capacities and the capacities of hydropower plants, of the Shih-Men, FeiTsui, and Kao-Tai reservoirs are shown in Table V.

Inflow data. The historical flow of the driest year was considered in this paper. The 10-day (the traditional reservoir operation period) magnitudes of the inflow from June 1964 to May 1965 are shown in Figure 10.

Water supply targets. The water supply target year is set to be 2021. These targets include public water and irrigation of the Shih-Men reservoir, shown in Figures 11-13. Figure 11 shows the public water targets of the Shih-Men, Tao-Yuan and Pan-Shin plants, which are located downstream of the Shih-Men reservoir (see Figure 9). Figure 12 shows the public water targets of the Pan-Shin plant, which is supported from the Taipei area. Figure 13 shows the irrigation targets of the Shih-Men reservoir.

Table V. Major properties of reservoirs in Tan-Shui River basin

\begin{tabular}{lccc}
\hline Reservoir & $\begin{array}{c}\text { Dam height } \\
(\mathrm{m})\end{array}$ & $\begin{array}{c}\text { Total storage } \\
\left(10^{6} \mathrm{~m}^{3}\right)\end{array}$ & $\begin{array}{c}\text { Hydropower plant } \\
\text { capacity }(\mathrm{kW})\end{array}$ \\
\hline Shih-Men & 133 & $250 \cdot 02$ & $45000 \times 2$ \\
Fei-Tsui & $122 \cdot 5$ & $390 \cdot 2$ & 70000 \\
Kao-Tai & 185 & $211 \cdot 3$ & 50000 \\
\hline
\end{tabular}

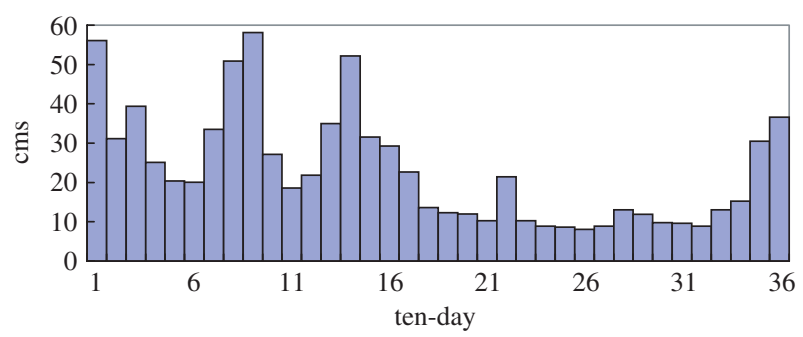

Figure 10. Inflow data of Shih-Men reservoir in the driest year, 1964 


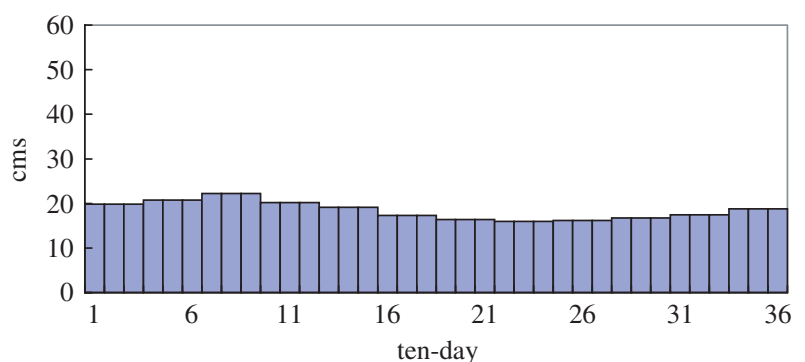

Figure 11. Public water demands of Shih-Men reservoir

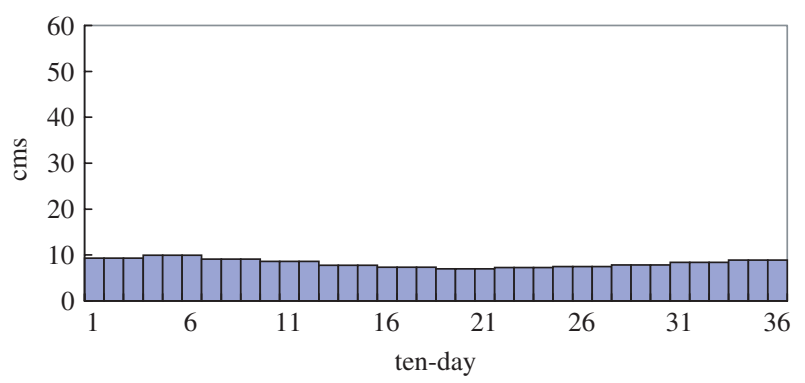

Figure 12. Public water demands of Pan-Shin plant (support from Fei-Tsui reservoir)

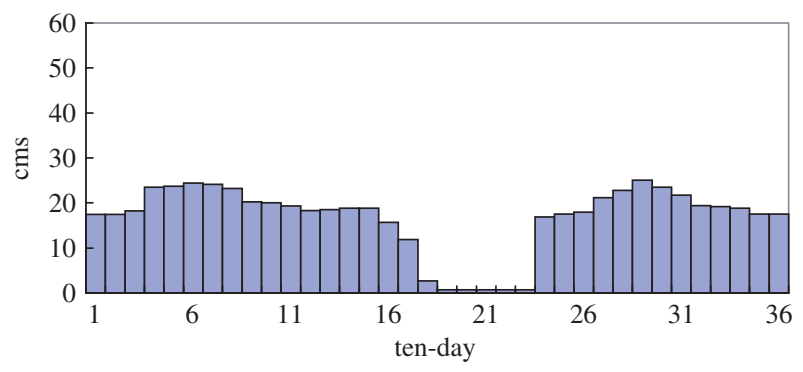

Figure 13. Irrigation demands of Shih-Men reservoir

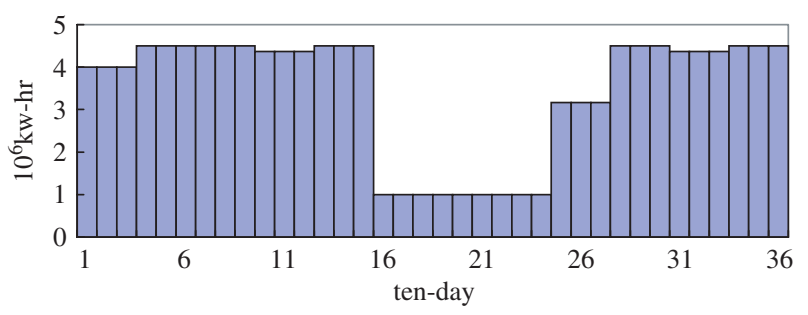

Figure 14. Hydropower generation targets of Shih-Men reservoir

Hydropower targets. The release of a reservoir can be transformed to hydropower generation using the powerto-discharge ratio $\left(\mathrm{kW} \mathrm{CMS}^{-1}\right)$ shown in Table VI. The hydropower generation contracts (see Figure 14) with the hydropower plant are used to make the hydropower targets:

$$
\text { Hydropower target }=\frac{\mathrm{kW}-\mathrm{h} \text { of contract }}{24 \mathrm{~h} \times \text { ratio }\left(\mathrm{kW} \mathrm{CMS}^{-1}\right)}
$$

\section{Applying the hypercubic distributed genetic algorithm}

Decision variables coding. Coding components of possible solutions into a chromosome is the first part of a GA formulation. Each chromosome is a potential solution and is comprised of a series of substrings or genes, representing components or variables that can be used to evaluate the objective function of the problem. The solutions to the problems described above were obtained by using both the conventional GA and the HDGA. The chromosome length may be expressed as a product of the number of stages $(t=36)$ and number of reservoirs $(i=1,2)$ in the system. The decision variables are releases during each time period $R_{i}(t), t=1, \ldots, 36$. Each real-coded gene within the chromosome represents a release made from the reservoir and can take up any value between the upper and lower bounds of releases.

Objective function. The objective function of the HDGA is to minimize the deviations from the hydropower and water supply targets of the Shih-Men area, because a water deficit always occurs in Shih-Men operation and Fei-Tsui has much surplus water lacking effective utilization. It can be written as

$$
\begin{gathered}
\operatorname{minimize} \sum_{t=1}^{36}\left[W_{\mathrm{hp}}\left(\mathrm{HPTG}_{t}-\mathrm{HP}_{t}\right)^{2}\right. \\
\left.+W_{\mathrm{ws}}\left(\mathrm{WSTG}_{t}-\mathrm{WS}_{t}\right)^{2}\right]
\end{gathered}
$$

where HPTG is the hydropower target of the Shih-Men area, HP is the hydropower generated, WSTG is the public water and irrigation demand targets in the ShihMen area, and WS is the water supply releases to be determined by an optimal method. Time steps are set to be 3610-day periods from June in one year to May in the next year. $W_{\mathrm{hp}}$ and $W_{\mathrm{ws}}$ represent the related weights of the hydropower and water supply targets respectively and were set to unity.

Constraints. The water balance of a multi-reservoir system is considered as the system constraint.

\section{Kao-Tai reservoir}

$$
R_{t}(1)=S_{t-1}(1)-S_{t}(1)+\mathrm{QK}_{t}-\mathrm{EVA}_{t}(1)
$$

\section{Shih-Men reservoir}

$$
R_{t}(2)=S_{t-1}(2)-S_{t}(2)+\mathrm{QLKS}_{t}+R_{t}(1)-\mathrm{EVA}_{t}(2)
$$

where $R_{t}(i), i=1,2$, is the release of each reservoir at time $t \quad(i=1$ : Kao-Tai reservoir; $i=2$ : Shih-Men reservoir), $S_{t}(i), i=1,2$, is the initial storages of each reservoir at time $t, S_{t+1}(i), i=1,2$, is the initial storage of each reservoir at time $t+1, \operatorname{EVA}_{t}(i), i=1,2$, is the

Table VI. Characteristics of the hydro-power plant in Shih-Men reservoir

\begin{tabular}{llllllllll}
\hline Water level $(\mathrm{m})$ & 245 & 240 & 235 & 225 & 220 & 215 & 210 & 200 & 195 \\
Hydropower constants $\left(\mathrm{kW} \mathrm{cm}{ }^{-1} \mathrm{~s}^{-1}\right)$ & $957 \cdot 6$ & $920 \cdot 1$ & 989.9 & 775.9 & $715 \cdot 4$ & 668.9 & $619 \cdot 6$ & $508 \cdot 0$ & $443 \cdot 9$ \\
\hline
\end{tabular}


evaporations of each reservoir at time $t, \mathrm{QK}_{t}$ is the inflow of Kao-Tai reservoir at time $t$, and $\mathrm{QLKS}_{t}$ is the lateral flow between the Kao-Tai and Shih-Men reservoirs.

The other constraints are as follows. Water levels at any period must be higher than the minimum level (intake elevations) and below the flood control level or other limitations. All diversion facility and power-plant equipment capacity limitations in the system must be satisfied. Therefore, the system is subject to constraints expressed as follows:

$$
\begin{aligned}
& S_{i, \min } \leq S_{i}(t) \leq S_{i, \max } \\
& R_{i, \min } \leq R_{i}(t) \leq R_{i, \max }
\end{aligned}
$$

where $S_{i, \min }$ and $S_{i, \max }(i=1,2)$ are respectively the allowable minimum and maximum storages in reservoir $i$ and $R_{i, \min }$ and $R_{i, \max }(i=1,2)$ are respectively the lower and upper bounds on the releases from reservoir $i$.

\section{Results and discussions}

The conventional GA involves three genetic operators: linear ranking selection with $\eta_{\min }=0.4$, blend- $\alpha=0.5$ crossover and the Gaussian mutation of a real-coded representation. We set the population size as 100 and ran with a fixed length of 5000 generations for case 1 and 1500 generations for case 2. In the HDGA, the size of each subpopulation is set to be 50; Table I presents the values of other parameters, such as $\eta_{\min }$ and $\alpha$ values for various subpopulations.

The optimal solution was obtained within 1000 generations by using the HDGA. The search space covers 400000 evaluations. The optimal objective value is $64603 \cdot 4$, and the total water deficit is $116 \cdot 8 \times 10^{6} \mathrm{~m}^{3}$. Figure 15 plots the storage of the Shih-Men reservoir in each operating period in case 1 (excluding Kao-Tai reservoir).

All subpopulations converge in fewer than 300 generations; the search space thus includes 120000 evaluations. The achieved optimal objective value is 37497.7 and the total water deficit is $71.1 \times 10^{6} \mathrm{~m}^{3}$. Figure 16 shows the water storages of the Shih-Men reservoir in all 36 operating periods in case 2 . As we compare the water storages

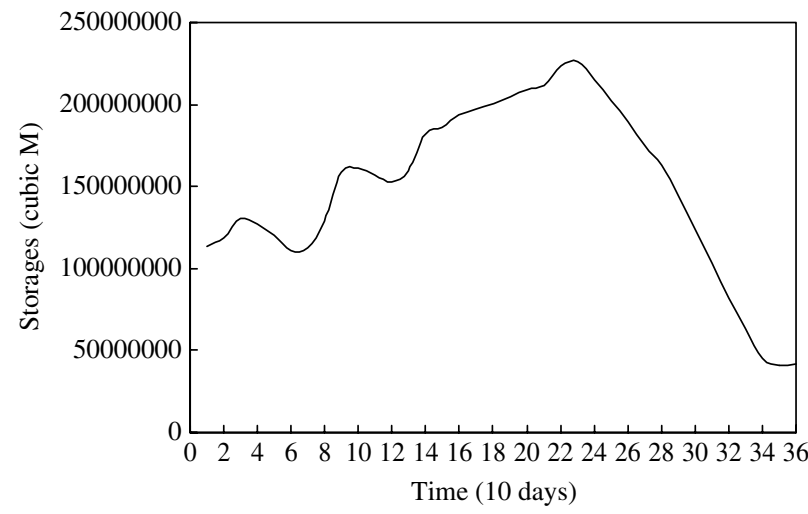

Figure 15. Storages of Shih-Men reservoir optimal operation (case 1)

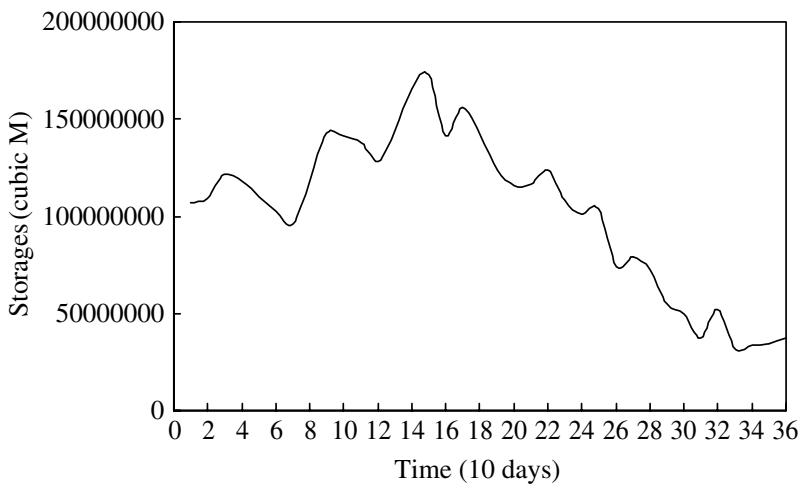

Figure 16. Storages of Shih-Men reservoir optimal operation (case 2)

Table VII. Comparisons between the two methods

\begin{tabular}{lcr}
\hline & Conventional GA & HDGA \\
\hline Case 1 objective value & $78486 \cdot 5$ & $64603 \cdot 4$ \\
Total deficit $\left(10^{6} \mathrm{~m}^{3}\right)$ & $145 \cdot 9$ & $116 \cdot 8$ \\
Case 2 objective value & 440937 & 37497.7 \\
Total deficit $\left(10^{6} \mathrm{~m}^{3}\right)$ & $85 \cdot 4$ & $71 \cdot 1$ \\
\hline
\end{tabular}

of case 1 with case 2 (Figures 15 and 16), it is easy to see that the storages of the Shih-Men reservoir in all operating periods in case 2 are fewer than those of case 1 . This is mainly because water could be stored in the Kao-Tai reservoir for case 2 .

Table VII shows the optimal results of the conventional GA and the HDGA for both cases. It appears that the conventional GA has higher objective values (with higher total deficits) than the HDGA for both cases. The results demonstrate that the conventional GA often converges prematurely and the HDGA outperforms it in these cases considered. The water deficit is shown to be low, when Kao-Tai reservoir is included in the Shih-Men reservoir system (case 2). Accordingly, the proposed Kao-Tai reservoir should be constructed to distribute water during a drought.

\section{CONCLUSIONS}

A hypercubic-topology-based HDGA is proposed, in which the subpopulations of the front side use different crossover operators with exploration and those from the rear side use crossover operators with exploitation. The extent of exploration or exploitation of the crossover operators applied to the subpopulations on the same side is gradual, yielding parallel multi-resolution with regard to the crossover operator. The algorithm of parallel structures used herein could significantly reduce the number of computations and evaluations and enhance the possibility of obtaining the optimal solution.

In the four-reservoir problem, a global optimal solution was easily reached from all subpopulations. The results indicate that the application of the HDGA to multiple-reservoir system problems is efficient and that the conventional GA cannot easily reach the optimal solution within a specified time period. The HDGA, with 
these revised operators, solves the problem significantly more effectively, and could be easily generalized to other highly nonlinear systems.

The proposed method was evaluated by application to an actual operational record and was tested under two conditions. The optimal solution for the Tan-Shui River basin, Taiwan, obtained using the HDGA is better than that obtained with the conventional GA. Owing to the conventional GA often converging prematurely, the HDGA outperforms it in these cases considered. This demonstrates that the HDGA has lower objective values (with lower total deficits) than the conventional GA within the same computing time. The results also indicate that the HDGA can be efficiently applied to large finitehorizon multi-reservoir system problems, in which the objective function is complex and the other optimization methods are difficult to apply.

If an effective prediction model of inflow were to be constructed, then the real-time reservoir operation with optimal releases obtained by the HDGA would be more successful in a drought warning situation. Future work will include a real-time operation model to decrease the water deficit.

\section{REFERENCES}

Antonisses HJ. 1989. A new interpretation of schema notation that overturns the binary encoding constraint. In Proceedings of the Third International Conference on Genetic Algorithms. Morgan Kaufmann: San Mateo, CA; 86-91.

Baker JE. 1985. Adaptive selection methods for genetic algorithms. In Proceedings of the 1st International Conference on Genetic Algorithms, Grefenstette JJ (ed.). Lawrence Erlbaum: Hillsdale, NJ; 101-111.

Bower TA, Hufschmidt MM, Reedy WW. 1962. Operating procedures: their role in design of water resources systems by simulation analysis. In Design of Water Resources Systems, Maass A (ed.). Harvard University Press: Cambridge, MA; 440-460.

Cai X, McKinney DC, Lasdon L. 2001. Solving nonlinear water management models using a combined genetic algorithm and linear programming approach. Advances in Water Resources 24(6): 667-676.

Cantu-Paz E. 1995. A summary of research on parallel genetic algorithms. University of Illinois at Urbana-Champaign, Illinois Genetic Algorithms Laboratory, IlliGAL Rep. 95007.

Chang F-J, Chen L. 1998. Real-coded genetic algorithm for rule-based flood control reservoir management. Water Resources Management 12: 185-198.

Chang F-J, Lai J-S, Kao L-S. 2003. Optimization of operation rule curves and flushing schedule in a reservoir. Hydrological Processes 17: $1623-1640$.

Chang F-J, Chen L, Chang L-C. 2005. Optimizing the reservoir operation rule curves by genetic algorithms. Hydrological Processes 19: 2277-2289

Chen L. 2003. Real-coded genetic algorithm optimization of longterm reservoir operation. Journal of the American Water Resources Association 39(5): 1157-1165.

Collins RJ. 1992. Studies in artificial evolution. PhD dissertation, University of California, Los Angeles.

Davidor YA. 1991. Naturally occurring niche species phenomenon: the model and first results. In Proceedings of the 4th International Conference on Genetic Algorithms, Belew R, Booker LB (eds). Morgan Kaufmann: San Mateo, CA; 257-263.

Davis L. 1991. Hybridization and numerical representation. In The Handbook of Genetic Algorithms, Davis L (ed.). Van Nostrand Reinhold: New York; 61-71.

Esat V, Hall MJ. 1994. Water resources system optimization using genetic algorithms. In Proceedings, 1st International Conference on Hydroinformatics. Balkema: Rotterdam, The Netherlands; 225-231.

Eshelman LJ, Schaffer JD. 1991. Preventing premature convergence in genetic algorithms by preventing incest. In Proceedings of the 4th
International Conference on Genetic Algorithms, Belew R, Booker LB (eds). Morgan Kaufmann: San Mateo, CA; 115-122.

Eshelman LJ, Schaffer JD. 1993. Real-coded genetic algorithms and interval-schemata. In Foundations of Genetic Algorithms 2, Whitley LD (ed.). Morgan Kaufmann: San Mateo, CA; 187-202.

Erickson JA, Smith RE, Goldberg DE. 1991. SGA-Cube, a simple genetic algorithm for nCUBE 2 hypercube parallel computers. TCGA Report No. 91005, The University of Alabama.

Goldberg DE. 1989. Genetic Algorithms in Search, Optimization and Machine Learning. Addison Wesley: Reading, MA.

Goldberg DE. 2000. Designing a competent simple genetic algorithm for search and optimization. Water Resources Research 36(12): 3757-3761.

Goldberg DE, Deb K. 1991. A comparative analysis of selection schemes used in genetic algorithms. In Foundations of Genetic Algorithms, Rawlins G (ed.). Morgan Kaufmann: 69-93.

Grefenstette JJ. 1986. Optimization of control parameters for genetic algorithms. IEEE Transactions on Systems, Man, and Cybernetics SMC-16(1): 122-128.

Heidari M, Chow VT, Kokotovic PV, Meredith DD. 1971. Discrete differential dynamic programming approach to water resources systems optimization. Water Resources Research 7(2): 273-282.

Herrera F, Lozano M. 1997. Heterogeneous distributed genetic algorithms based on the crossover operator. In 2nd IEE/IEEE International Conference on Genetic Algorithms in Engineering Systems: Innovations and Applications; 203-208.

Herrera F, Lozano M. 2000. Gradual distributed real-coded genetic algorithms. IEEE Transactions on Evolutionary Computation 4(1): $43-63$.

Hinterding R. 1995. Gaussian mutation and self-adaptation in numeric genetic algorithms. In IEEE International Conference on Evolutionary Computation. IEEE Press: 384-389.

Holland JH. 1975. Adaptation in Natural and Artificial Systems. The University of Michigan Press: Ann Arbor, MI

Jairaj PG, Vedula S. 2000. Multireservoir system optimization using fuzzy mathematical programming. Water Resources Management 14: 457-472.

Karpouzos DK, Delay F, Katsifarakis KL, de Marsily G, 2001. A multipopulation genetic algorithm to solve the inverse problem in hydrogeology. Water Resources Research 37(9): 2291-2302.

Larson RE. 1968. State Increment Dynamic Programming. Elsevier: New York.

Man KF, Tang KS, Kwong S. 1999. Generic Algorithms. City University of Hong Kong.

Michalewicz Z. 1992. Genetic Algorithms + Data Structures = Evolution Programs. Springer: New York.

Muhlenbein H, Schomisch M, Born J. 1991. The parallel genetic algorithm as function optimizer. In Proceedings of the 4th International Conference on Genetic Algorithms, Belew R, Booker LB (eds). Morgan Kaufmann: San Mateo, CA; 271-278.

Mujumdar PP, Narulkar S. 1992. Optimization models for multireservoir planning and operation. Jalvigyan Sameeksha VIII(1): 29-52.

Oliveira R, Loucks DP. 1997. Operating rules for multi reservoir systems. Water Resources Research 33(4): 839-852.

Renders J-M, Flasse SP. 1996. Hybrid methods using genetic algorithms for global optimization. IEEE Transactions on Systems, Man, and Cybernetics SMC-26(2): 243-258.

Sharif M, Wardlaw R. 2000. Multireservoir system optimization using genetic algorithms: case study. Journal of Computing in Civil Engineering, ASCE 14(4): 255-263.

Tanese R. 1987. Parallel genetic algorithms for a hypercube. In Proceedings of the 2nd International Conference on Genetic Algorithms and Their Applications, Grefenstette JJ (ed.). Lawrence Erlbaum: Hillsdale, NJ; 177-183.

Tanese R. 1989. Distributed genetic algorithms. In Proceedings of the 3rd International Conference on Genetic Algorithms, Schaffer JD (ed.). Morgan Kaufmann: San Mateo, CA; 434-439.

Wardlaw R, Sharif M. 1999. Evaluation of genetic algorithms for optimal reservoir system operation. Journal of Water Resources Planning and Management, ASCE 125(1): 25-33.

Wright A. 1991. Genetic algorithms for real parameter optimization. In Foundations of Genetic Algorithms, Rawlins GJE (ed.). Morgan Kaufmann: San Mateo, CA; 205-218.

Wurbs RA. 1993. Reservoir-system simulation and optimization models. Journal of Water Resources Planning and Management, ASCE 119(4): $455-472$.

Yeh WW-G. 1985. Reservoir management and operation models: a state-of-the-art review. Water Resources Research 21(12): 1797-1818. 